\section{DIFFERENTIATION SUBGROUPS WITHIN LKB1-DEFICIENT LUNG CANCER INFLUENCE BOTH THE IMMUNE EXCLUSION PHENOTYPE AND CELLULAR COMPOSITION OF THE IMMUNE MICROENVIRONMENT}

${ }^{1}$ Jacob Kaufman*, ${ }^{2}$ Doug Cress, ${ }^{2}$ Theresa Boyle, 'David Carbone, ${ }^{3}$ Neal Ready, Kris Wood. ${ }^{1}$ Ohio State University, Columbus, OH, USA; ${ }^{2}$ Moffitt Cancer Center, Tampa, FL, USA; ${ }^{3}$ Duke University Medical Center, Chapel Hill, NC, USA

Background LKB1 (STK11) is a commonly disrupted tumor suppressor in NSCLC. Its loss promotes an immune exclusion phenotype with evidence of low expression of interferon stimulated genes (ISG) and decreased microenvironment immune infiltration. ${ }^{1}{ }^{2}$ Clinically, LKB1 loss induces primary immunotherapy resistance. ${ }^{3}$ LKB1 is a master regulator of a complex downstream kinase network and has pleiotropic effects on cell biology. Understanding the heterogeneous phenotypes associated with LKB1 loss and their influence on tumor-immune biology will help define and overcome mechanisms of immunotherapy resistance within this subset of lung cancer.

Methods We applied multi-omic analyses across multiple lung adenocarcinoma datasets ${ }^{2}{ }^{4-6}$ (>1000 tumors) to define transcriptional and genetic features enriched in LKB1-deficient lung cancer. Top scoring phenotypes exhibited heterogeneity across LKB1-loss tumors, and were further interrogated to determine association with increased or decreased markers of immune activity. Further, immune cell-types were estimated by Cibersort to identify effects of LKB1 loss on the immune microenvironment. Key conclusions were confirmed by blinded pathology review.

Results We show that LKB1 loss significantly affects differentiation patterns, with enrichment of ASCL1-expressing tumors with putative neuroendocrine differentiation. LKB1-deficient neuroendocrine tumors had lower expression of Interferon Stimulated Genes (ISG), MHC1 and MHC2 components, and immune infiltration compared to LKB1-WT and non-neuroendocrine LKB1-deficient tumors (figure 1).The abundances of 22 immune cell types assessed by Cibersort were compared between LKB1-deficient and LKB1-WT tumors. We observe skewing of immune microenvironmental composition by LKB1 loss, with lower abundance of dendritic cells, monocytes, and macrophages, and increased levels of neutrophils and plasma cells (table 1). These trends were most pronounced among tumors with neuroendocrine differentiation, and were concordant across three independent datasets. In a confirmatory subset of 20 tumors, plasma cell abundance was assessed by a blinded pathologist. Pathologist assessment was 100\% concordant with Cibersort prediction, and association with LKB1 loss was confirmed $(\mathrm{P}=0.001)$.

Conclusions We conclude that tumor differentiation patterns strongly influence the immune microenvironment and immune exclusion characteristics of LKB1-deficient tumors. Neuroendocrine differentiation is associated with the strongest immune exclusion characteristics and should be evaluated clinically for evidence of immunotherapy resistance. A novel observation of increased plasma cell abundance is observed across multiple datasets and confirmed by pathology. Causal mechanisms linking differentiation status to immune activity is not well understood, and the functional role of plasma cells in the immune biology of LKB1-deficient tumors is undefined. These questions warrant further study to inform precision immuno-oncology treatments for these patients.

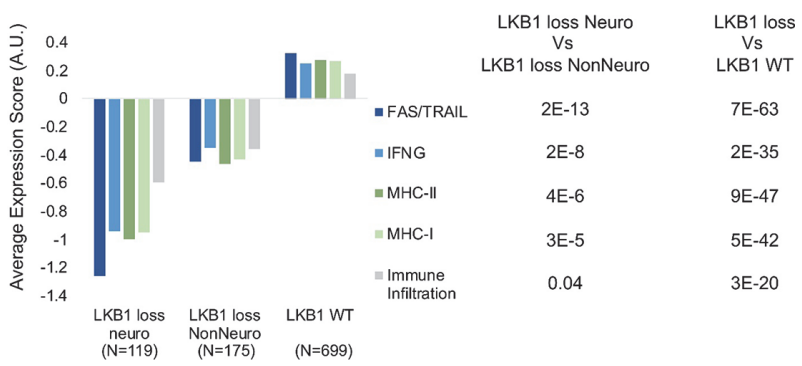

Abstract 909 Figure 1 Immune-associated Gene Expression Profiles Affected by Neuroendocrine Differentiation within LKB1-Deficient Lung Adenocarcinomas. Gene expression profiles corresponding to five immune-associated phenotypes are shown with bars indicating average GEP scores for tumors grouped according to LKB1 and neuroendocrine status as indicated. P-values represent results from Student's T-test between groups as indicated.

Abstract 909 Table 1 LKB1 Loss Affects Composition of Immune Microenvironment. Values indicate log10 P-values comparing LKB1loss to LKB1-WT tumors. Positive (red) indicates increased abundance in LKB1 loss. Negative (blue) indicates decreased abundance.

\begin{tabular}{|c|c|c|c|}
\hline \multicolumn{1}{|c|}{} & TCGA & Moffitt & Affy Array \\
\cline { 2 - 4 } B cells naive & 3.5 & -0.2 & -0.1 \\
\hline B cells memory & -0.4 & -0.5 & -0.8 \\
\hline Plasma cells & $\underline{15.6}$ & $\underline{16.9}$ & $\underline{\mathbf{6 . 8}}$ \\
\hline T cells CD8 & 0.6 & 1 & 0 \\
\hline T cells CD4 naive & 0.9 & -0.4 & -0.3 \\
\hline T cells CD4 memory resting & -1.2 & 0.3 & -0.3 \\
\hline T cells CD4 memory activated & 0 & 0.9 & 0.7 \\
\hline T cells follicular helper & 3 & 2.7 & -0.2 \\
\hline T cells regulatory (Tregs) & -1.5 & -0.5 & 0 \\
\hline T cells gamma delta & -0.6 & -2.1 & -0.7 \\
\hline NK cells resting & 1.5 & 1.1 & 1.8 \\
\hline NK cells activated & 2.3 & 0.8 & -0.5 \\
\hline Monocytes & $\underline{\mathbf{2 . 7}}$ & $\underline{\mathbf{- 1 . 7}}$ & $\underline{\mathbf{- 2 . 1}}$ \\
\hline Macrophages M0 & 0 & -0.2 & -0.2 \\
\hline Macrophages M1 & $\underline{\mathbf{3 . 9}}$ & $\underline{\mathbf{- 2 . 3}}$ & $\underline{\mathbf{- 2 . 4}}$ \\
\hline Macrophages M2 & -5 & 0.1 & -5.1 \\
\hline Dendritic cells resting & $\underline{-6}$ & $\underline{-4.7}$ & $\underline{\mathbf{5 . 2}}$ \\
\hline Dendritic cells activated & $\mathbf{2 . 7}$ & 0.8 & 0.3 \\
\hline Mast cells resting & $\underline{\mathbf{3 . 9}}$ & $\underline{-4.4}$ & $\underline{\mathbf{- 5 . 9}}$ \\
\hline Mast cells activated & 0 & -0.1 & 0.3 \\
\hline Eosinophils & -0.1 & -0.7 & -0.8 \\
\hline Neutrophils & $\underline{\mathbf{3 . 9}}$ & $\underline{\mathbf{8 . 4}}$ & $\underline{\mathbf{2 . 4}}$ \\
\hline
\end{tabular}

Acknowledgements This work was funded by SITC AZ Immunotherapy in Lung Cancer grant (SPS256666) and DOD Lung Cancer Research Program Concept Award (LC180633).

\section{REFERENCES}

1. Skoulidis F, Byers LA, Diao L, et al. Co-occurring genomic alterations define major subsets of KRAS-mutant lung adenocarcinoma with distinct biology, immune profiles, and therapeutic vulnerabilities. Cancer Discov 2015;5:860-77.

2. Schabath MB, Welsh EA, Fulp WJ, et al. Differential association of STK11 and TP53 with KRAS mutation-associated gene expression, proliferation and immune surveillance in lung adenocarcinoma. Oncogene 2016;35:3209-16.

3. Skoulidis F, Goldberg ME, Greenawalt DM, et al. STK11/LKB1 mutations and PD1 inhibitor resistance in KRAS-mutant lung adenocarcinoma. Cancer Discovery 2018:8:822-835.

4. Cancer Genome Atlas Research Network. Comprehensive molecular profiling of lung adenocarcinoma. Nature 2014;511:543-50. 
5. Chitale D, Gong Y, Taylor BS, et al. An integrated genomic analysis of lung cancer reveals loss of DUSP4 in EGFR-mutant tumors. Oncogene 2009;28:2773-83.

6. Shedden K, Taylor JM, Enkemann SA, et al. Gene expression-based survival prediction in lung adenocarcinoma: a multi-site, blinded validation study. Nat Med 2008;14:822-7.

http://dx.doi.org/10.1136/jitc-2021-SITC2021.909 